\title{
BMJ Open Evaluation of instructions in patient information leaflets for the use of intranasal corticosteroid sprays: an observational study
}

Corine Rollema, ${ }^{1,2}$ Eric M van Roon, ${ }^{1,2}$ Anne GM Schilder, ${ }^{3}$ Tjalling W de Vries $^{4}$

To cite: Rollema C, van Roon EM, Schilder AGM, et al. Evaluation of instructions in patient information leaflets for the use of intranasal corticosteroid sprays: an observational study. BMJ Open 2019;9:e026710. doi:10.1136/ bmjopen-2018-026710

- Prepublication history for this paper is available online. To view these files, please visit the journal online (http://dx.doi. org/10.1136/bmjopen-2018026710).

Received 28 September 2018 Revised 11 November 2018 Accepted 22 November 2018

Check for updates

(c) Author(s) (or their employer(s)) 2019. Re-use permitted under CC BY-NC. No commercial re-use. See rights and permissions. Published by BMJ.

${ }^{1}$ Department of Clinical Pharmacy and Pharmacology, Medisch Centrum Leeuwarden, Leeuwarden, Netherlands ${ }^{2}$ Department PharmacoTherapy, -Epidemiology and -Economy, Groningen Research Institute of Pharmacy, University of Groningen (RUG), Groningen, Netherlands

${ }^{3}$ evidENT, Ear Institute, University College London, UCLH Royal National Throat, Nose and Ear Hospital, London, UK

${ }^{4}$ Department of Paediatrics, Medical Center Leeuwarden, Leeuwarden, Netherlands

Correspondence to

Corine Rollema;

corinerollema@gmail.com

\section{ABSTRACT}

Objectives In this study, we analysed patient information leaflets (PILs) of intranasal corticosteroid sprays (INCS) of different manufacturers in the UK to determine if instructions for the use of INCS are complete and uniform. Setting PILs of all INCS of all manufacturers, available for patients in the UK, were collected from the British National Formulary website and the Medicines and Healthcare products Regulatory Agency website. All instructions in these PILs were analysed.

Participants We identified PILs of INCS from 21 different manufacturers, available for patients in the UK.

Results We analysed the instructions for the use of INCS in 21 different PILs and there is large variation in the PIL instructions for the technique of using INCS across PILs. Conclusion Complete and uniform instructions for the use of INCS are lacking in PILs for registered preparations in the UK. Structured and standardised instructions to be used by both professionals and patients are essential in order to optimise daily use of INCS.

\section{INTRODUCTION}

Allergic rhinitis (AR) is a common condition in both children and adults, with prevalences ranging from $9 \%$ in children aged $6-7$ years and $27 \%$ in adults aged 22-44years. ${ }^{12}$ The recommended pharmacological treatment of AR includes antihistamines and intranasal corticosteroids. Antihistamines are used in oral and intranasal dosage forms. Corticosteroids suppress inflammation and are available in oral dosage forms, but due to systemic adverse events (AEs), intranasal corticosteroids are preferred. ${ }^{34}$ Intranasal corticosteroids are available in sprays or drops and in most patients sprays are prescribed. An adequate technique of using intranasal corticosteroid sprays (INCS) is imperative: the technique may affect efficacy, AEs and compliance. ${ }^{5}$ Little data are available on the association between the quality of the techniques of using INCS and its safety and efficacy.

To our knowledge no standardised protocols are available with instructions for use of
Strengths and limitations of this study

- This observational study evaluates all patient information leaflets (PILS) of intranasal corticosteroid sprays (INCS) available for patients in the UK.

- This is the first study that evaluates PILs of INCS on uniformity and completeness.

- All PILs are collected from the British National Formulary and Medicines and Healthcare products Regulatory Agency websites and each PIL is checked whether a specific instruction for use of the INCS is included.

- We do not know if administration of INCS depends on the instruction described in PILS.

- We did not collect data on instructions for the use of INCS given by doctors, nurses and pharmacists and whether the PILs are used during these instructions.

INCS. Patient information leaflets (PILs) are the only source of information about instructions, in addition to the instructions given by the doctor, nurse or pharmacist. We explored which instructions for the use of INCS are provided in PILs by the different manufacturers in the UK.

\section{METHODS}

PILs of all INCS of all manufacturers, available for patients in the UK, were collected from the British National Formulary website and the Medicines and Healthcare products Regulatory Agency website. These PILs are identical to what is provided to the patient in the medicine pack. The instructions included in these PILs were categorised in the consecutive steps before, during and after administration of the INCS, as shown in table 1. For every PIL we evaluated whether it includes these steps and for each instruction we counted in how many of the PILs it was included. Missing instructions regarding before, during and after administration were coded as such and counted. Steps for priming and cleaning of 


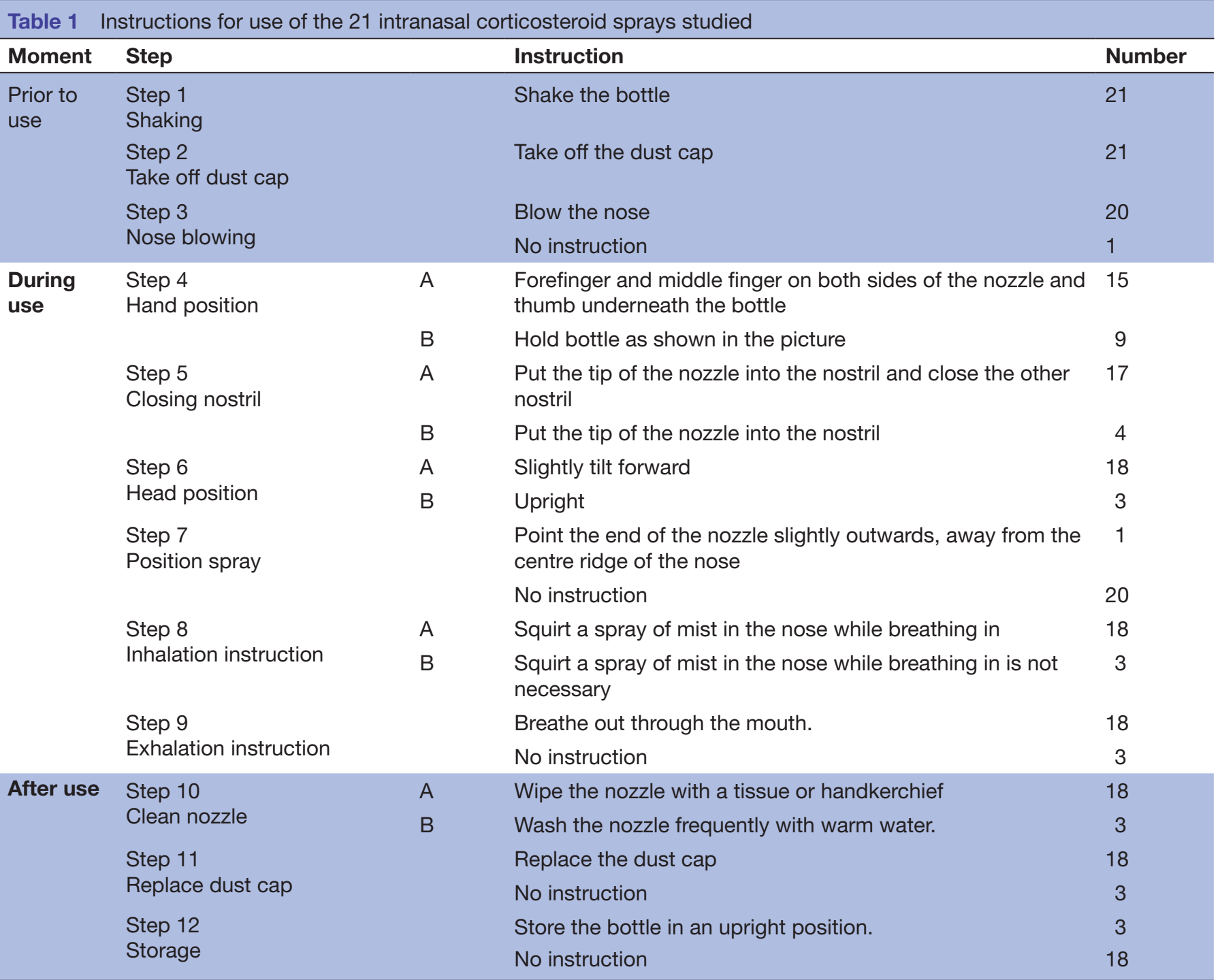

Described are the consecutive steps before, during and after use of INCS, the specific instruction for a step and the number of PILs wherein the specific instruction is described. Daily administration of INCS consists of 12 steps and for some steps different instructions are described.

the sprays are also mentioned in PILs of INCS, which can also be distinguished in consecutive steps. In this analysis, we only exemplify all steps for daily administration of INCS as mentioned in the PILs.

\section{Patient and public involvement}

Patients and public were not directly involved in this study.

\section{RESULTS}

We identified PILs of INCS from 21 different manufacturers (table 2). Table 1 shows how many PILs include instructions for each step, and table 2 shows an overview of each spray and whether the instruction is supplied. All PILs instruct to shake the bottle (step 1) and take the dust cap off (step 2) prior to use. Instructions for nose blowing, hand positioning, closing the nostril, head positioning, positioning of the spray, inhaling and exhaling, cleaning the nozzle, replacing the dust cap and storing of the spray (step 3 to step 12) varied or were not included.

In all 21 PILs one or more steps were missing. Instructions for nose blowing, positioning of the spray, exhaling, replacing the dust cap and storing were missing from 1 , 20, 3, 3 and 18 PILs, respectively. Instructions regarding hand positioning, closing the nostril, head positioning, inhaling and cleaning were conflicting across PILs (see table 1).

\section{DISCUSSION}

This study shows that there is a large variation in the PIL instructions for the technique of using INCS across PILs of those sprays available for UK patients.

There has been little research on consistency and impact of instructions for use of INCS given by the doctor, nurse or pharmacist on patients' use of these sprays and 


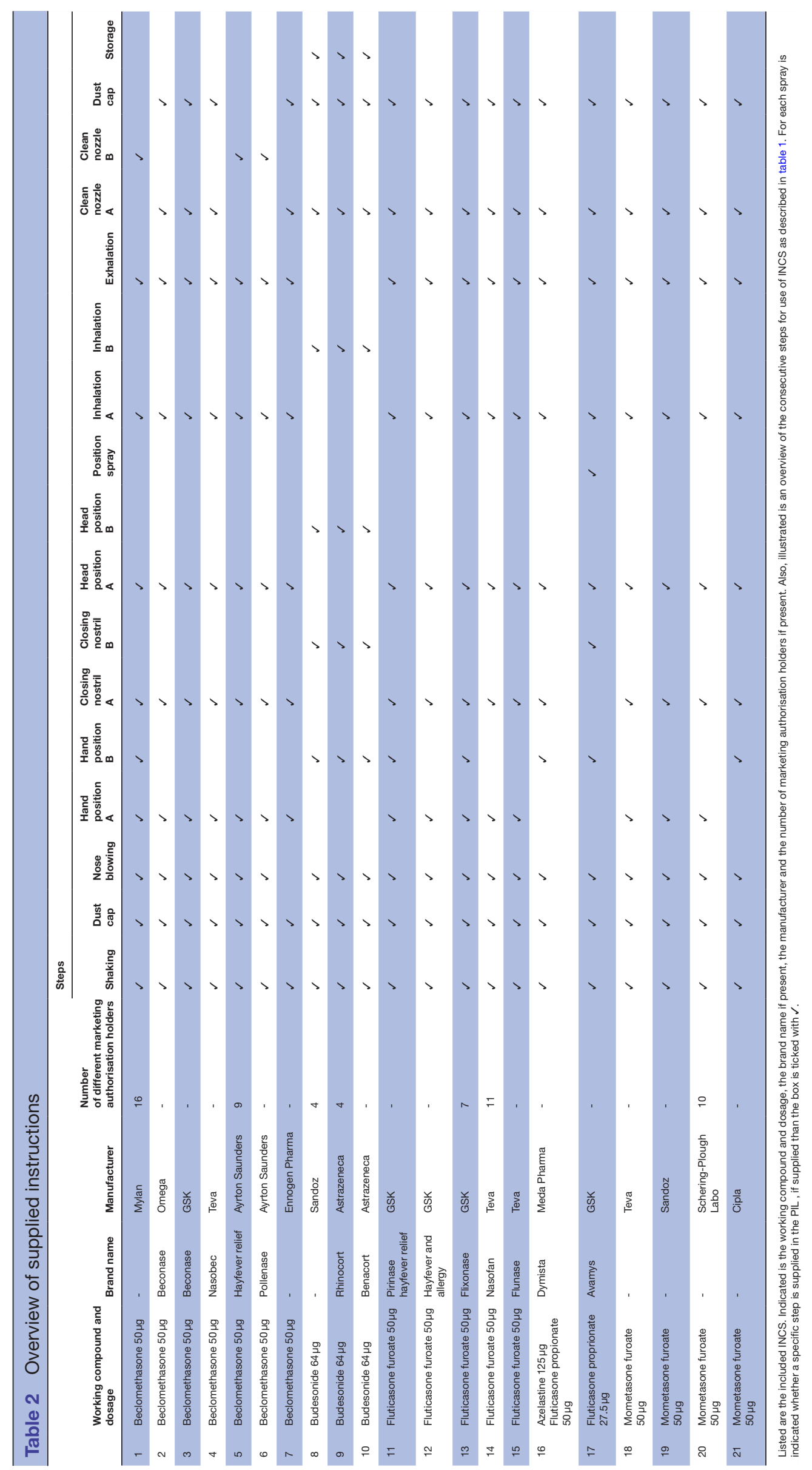

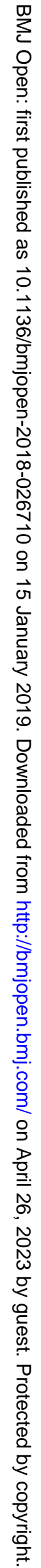


their clinical effectiveness. Nor has there been research on the role of PILs on these outcomes.

PILs fulfil a supporting role; information in PILs improve patients' knowledge, but information needs to concise and precise and information needs to be accompanied by oral information. ${ }^{6}$ Different or missing instructions in PILs for the same product of different manufacturers can confuse the patient and healthcare professionals. Instructions for correct use of INCS is a joined task of doctor, nurse, pharmacist and manufacturer. We believe it is essential to provide uniform and complete instructions in PILs.

Benninger et al reviewed the literature on techniques of intranasal steroids and their efficacy and developed detailed instructions for administration of INCS, in order to reach highest efficacy and prevent side effects. ${ }^{7}$ They recommend to administer the nasal spray in a neutral head position, so to minimise spillage of the drug into the throat which may cause mucosal irritation and facilitate absorption of the drug via the gastrointestinal tract. To minimise the risk of epistaxis, a known AE of INCS, it is recommended to point the nozzle of the spray outward, away from the nasal septum. ${ }^{8}$

The set of instructions of Benninger et al provides a good basis for the development of uniform and complete instructions for the use of INCS.

A limitation of our study is that we did not collect data on instructions for the use of INCS given by doctors, nurses and pharmacists and whether the PILs are used as a guide during these instructions.

Furthermore, intranasal corticosteroids are also available in nasal drops. The instructions for administration of these intranasal corticosteroid drops differ from the instructions for administration of INCS and are therefore not included in this study.

In conclusion, complete and uniform instructions for the use of INCS are lacking in PILs for registered preparations in the UK. Patients sometimes have to change their INCS due to price differences, stock availability and, sometimes, efficacy. It is confusing that INCS have a completely different set of instruction. Structured and standardised instructions to be used by both professionals and patients are essential in order to optimise daily use of INCS in patients with AR.

Contributors The study was part of the master research project of CR. During this master research CR was supervised by EMvR and TWdV. The original study protocol was set up by CR, EMvR, AGMS and TWdV. The practical part of the study was carried out by CR, hereby EMvR, AGMS and TWdV fulfilled an advisory and supervisory role. The design of the manuscript was set up by all four authors and CR elaborated the design into this manuscript. EMvR, AGMS and TWdV fulfilled an advisory and supervisory role during the writing process.

Funding The authors have not declared a specific grant for this research from any funding agency in the public, commercial or not-for-profit sectors.

Competing interests None declared.

Patient consent for publication Obtained.

Provenance and peer review Not commissioned; externally peer reviewed.

Data sharing statement This manuscript contains all data used for the presented results (presented in the Tables). No additional unpublished data is available. The data is obtained from patient information leaflets (PILs) that are available for patients in the United Kingdom, these PILs can be found on the internet.

Open access This is an open access article distributed in accordance with the Creative Commons Attribution Non Commercial (CC BY-NC 4.0) license, which permits others to distribute, remix, adapt, build upon this work non-commercially, and license their derivative works on different terms, provided the original work is properly cited, appropriate credit is given, any changes made indicated, and the use is non-commercial. See: http://creativecommons.org/licenses/by-nc/4.0/.

\section{REFERENCES}

1. Aït-Khaled N, Pearce N, Anderson HR, et al. Global map of the prevalence of symptoms of rhinoconjunctivitis in children: the international study of asthma and allergies in childhood (isaac) phase three. Allergy 2009;64:123-48.

2. Bousquet PJ, Leynaert B, Neukirch F, et al. Geographical distribution of atopic rhinitis in the european community respiratory health survey I. Allergy 2008;63:1301-9.

3. Roberts G, Xatzipsalti M, Borrego LM, et al. Paediatric rhinitis: position paper of the european academy of allergy and clinical immunology. Allergy 2013;68:n/a-16.

4. Brozek JL, Bousquet J, Baena-Cagnani CE, et al. Allergic Rhinitis and its Impact on Asthma (ARIA) guidelines: 2010 revision. J Allergy Clin Immunol 2010;126:466-76.

5. Ganesh V, Banigo A, McMurran AEL, et al. Does intranasal steroid spray technique affect side effects and compliance? Results of a patient survey. J Laryngol Otol 2017;131:991-6.

6. Sustersic M, Gauchet A, Foote A, et al. How best to use and evaluate Patient Information Leaflets given during a consultation: a systematic review of literature reviews. Health Expect 2017;20:531-42.

7. Benninger MS, Hadley JA, Osguthorpe JD, et al. Techniques of intranasal steroid use. Otolaryngol Head Neck Surg 2004;130:5-24.

8. Chong LY, Head K, Hopkins C, et al. Intranasal steroids versus placebo or no intervention for chronic rhinosinusitis. Cochrane Database Syst Rev 2016;4:CD011996. 